\title{
Commentary of Sichuan Opera Music Reform' Research Literature in the Latter Half of the 20th Century
}

\author{
Shengdong Yue, Yutong Xuan
}

\author{
School of Music of Chengdu Normal University, Sichuan, China \\ School of Music of Chengdu Normal University, Sichuan, China
}

Key words: the latter half of the 20th Century, Sichuan opera music reform, commentary

\begin{abstract}
This paper has sorted out and analyzed the research literature of 'Sichuan opera music reform' in the latter half of the 20th century and it has made the expounding and evaluation from the aspects of reform necessity, reform formation,reform's concrete measures, reform summary and reform reflection etc., besides, it has also made the prospect of the research method and direction in the future on the basis of pointing out its disadvantages.

About the related document literature of Sichuan opera music reform in the latter half of 20th century, the author firstly has looked up the currently eyeable, publicly issued and published newspaper, journal and academic proceedings, which includes Sichuan Daily, Chengdu Daily, Chongqing Daily, Sichuan Opera Artistic Research, Sichuan News, Sichuan Opera Art, Drama and Film, Sichuan Opera Learning and Study, Music Construction Essays, Sichuan Opera Music Seminar Proceedings, Sichuan Opera Music Reform Anthology, Sichuan Opera Vocal Music Art Seminar Proceedings, etc., and he has collected the related literature with a number about over 150 . Apart from the above print publication, the author has also got about 70 supplementary literature in the network literature database of CNKI (China National Knowledge Infrastructure) etc., besides, the got document literature content can be classified into 3 types, which main content can be separately narrated as follows.
\end{abstract}

\section{Discussion on Whether the Sichuan Opera Needs to Reform and How to Reform After 'Theater Reform'}

In May. 5, 1951, the state council issued 'Instructions about Theater Reform Work', and put forward the task 'Personnel Reform, System Reform and Theater Reform' so that to make the 'Theater Reform' become the key part of government's culture art planning layout. The Sichuan opera at that time has the view that the main problem of Sichuan opera music is high-pitched tune(a kind of rhyme scheme of Chinese opera) and the high-pitched tune just is the simple singing and its musicality is not obvious, besides, its aria(music for voices in a Chinese opera) is simple and the sound of the gong and drum is too noisy etc., opinions, In the frequent exchange between China and other foreign countries, it has absorbed a lot of experience and skills, eventually, it has made the determination that take the reform by taking the high-pitched tune as the focus.

When the Sichuan opera theater of Sichuan province took the reform experiment performance in the places of Chengdu and Chongqing, it had taken the attempt of Gao Qiang plus accompaniment and different degrees of spectrum cavity, besides, it also had taken the musical reform with the bold idea of combing the high-pitched tune spectrum cavity with the mixed band accompaniment, for the individual, it had adopted the way of 'full tune with full following'. These reforms have caused the 
debate on the inheritance and reform of the 'high-pitched tune plus accompaniment' question, which has caused the 'sensation effect' with extensive social influence, further more, this debated has lasted to the early $1980 \mathrm{~s}$. Sichuan opera circle cares about the success or failure, gain or loss of their exploration and the audience from all walks of life have also expound their own opinions on the newspapers and magazines, besides, the party and government departments still hold the colloquium in a row and the main discussion points are focused on the problems of 'adding accompaniment into Gao Qiang or not' and the related vocal accompaniment, interlude and tune name exception making and the discussion pointcut is mainly taken from the macro level.

Ao Xueqi has concluded the characteristics of Sichuan opera music in his Several Problems of Sichuan Opera Musical Reform, on which basis, he has also put forward the necessity and directionality of Sichuan opera music reform. Ao Xueqi, Qiu Zhongpeng has pointed that in his Discussion of Sichuan Opera Music Reform, the Sichuan opera music reform should be taken from the four aspects of adding musical instrument accompaniment, vocal accompaniment reform, percussion music reform and new tune name innovation. Zheng Yinfei has put forward in his Discussion on the Accompaniment Problems of Sichuan Opera's High-pitched Music, that integrate the accompaniment into the trinity of Sichuan opera aria, vocal accompaniment and percussion music so as to form the quaternity pattern. Yu Shucun has put forward in What is the Essential Issue? that we must develop the reform work of Sichuan opera music on the basis of acknowledging and respecting the tradition. Li Kangsheng has put forward in his What is the problem of Sichuan opera music? that the keynote of reform should be 'first inheritance and second reform', and the repertory identification should not only be the script identification but ignore the attention to Sichuan opera music. Sichuan opera music reform should be divided into two stages, the experimental performances and stage performance, and in different stages, different standards and requirements can be put forward. Yao Zhongfu has expounded in his Bel Canto, Feeling Expression and Into Play, the methods and ways of improving the Sichuan opera singing levels from the aspects of strengthening voice training, improving literacy and paying attention to the attractive singing etc. Shamei has expounded in the Better to Illustrate by Using Facts and Discussion from Others to Horizontal Pull that the Sichuan opera singing should use scientific method to take the voice training and the relation between spectrum cavity, accompaniment and musical style inheritance, further more, he has also showed us her feelings and experience when he creates his first Sichuan opera Red Plum for the Man, which work is accompanied all by western musical instruments.

\section{Design Demonstration and Concrete Measures of Sichuan Opera Musical Reform}

The number of this part of literature is big and it not only includes the reform discussion of the aria, tune names, gong and drum and accompaniment band, but also includes the specific planning and design of different operas types--Kun, Gao, Hu, Tan, Deng and different repertoire--traditional opera, modern opera, new historical opera etc. As most of the literature authors are the insiders of Sichuan opera groups and professional institutions, their discussion lays particular stress on the micro level and has more practical significance.

Examples of developing discussion from the aspect of innovation and design include: Yangwei has put forward in the work Discussion of Sichuan Opera Music Design that on the basis of traditional pattern, it also needs to reflect the times features, only by fully absorbing the modern elements, can it be more better to use music to create characters, besides, the musical reform should take the aria as the center to drive the design of other aspects so that to achieve the unification of musical language. Jiahui, in The Normativity and Flexibility of Sichuan Opera's High-pitched Tune Name and Li Zhaohong, in The Continuing Use of Old Tune and The Reflection of Modern Life all 
put forward that the tune names should be used flexibly according to the script content and personal character, besides, people should shape the characters in the new period by using the realistic creation method so that to reflect the new life of socialism. Peng Chaoyi, in The Breakthrough and Development of Modern Sichuan Opera Comparing with the Traditional Music, has demonstrated the enriching and development of opera tune art from the aspects of folk song into play, mode change and polyphony application etc., besides, he has put forward the rhythm breakthrough according to the music plate changes and accompaniment technique innovation according to percussion music reform and multi-tone music application. Yang Yongfu, in Master of the Times' Pulse has expounded the solution for the problems of 'untruthfulness, slippage, simplicity' of Tan opera from the aspects of vocal cavity's combination of opera and songs, attempt of gong and drum playing breakthrough and reform of sound production ways etc. Zhoujun, in Exploration of Sichuan Opera's Tune Style, has put forward that the Sichuan opera tune is suitable for reflecting the modern life and comic themes, which especially complies with young audiences' aesthetic interest, so in the aspects of orchestra, percussion music setting, singing method and aria singing style and aria etc., the style and characteristics of light music should be taken as the reform direction.Discussion on the Horizontal Reference of Sichuan Opera High-pitched Tune in Modern Opera 'SisterJiang' written by Zhou Zhilin has certain degree of representativeness in the discussion of the transplant opera in Sichuan opera's modern opera, and it has expounded the necessity and specific methods for horizontal borrowing from three aspects of music plate, chorus and accompaniment, besides, it has showed the good changes brought by transplanting the new opera into the modern opera of Sichuan opera. The Important Breakthrough and Sharp Innovation written by Hanzheng and the High-Pitched Tune Music and Modern Opera of Sichuan Opera written by Rao Bingjun have discussed from the original pattern inheritance, reformed performance and Fan tune-modulation of Sichuan opera modern opera to the vocal cavity pattern innovation of female voice to male tune, two-part harmony, mixed chorus, male-female duo, trio, troll, accompaniment with lyrics, accompaniment without lyrics, climax progress etc., besides, they have concluded the success and failure, gain and loss of Sichuan opera high-pitched reform according to the specific performance effect of different repertoires. Wang Wenxun, in his works Discussion of the Horizontal Reference of Sichuan Opera Music Based on the Aria Design of 'Big Feet Lady' and Vertical Inheritance and Horizontal Reference has pointed that the music design not only needs to take vertical inheritance of China's traditional vocal cavity, but also needs to take horizontal reference of modern music, especially the western music, so as to open up a broad development space for Sichuan opera musical reform.

Sichuan opera high-pitched tune is the most representative part of Sichuan opera art and it is also the most perfect vocal cavity types, besides the discussion about its aria and vocal accompaniment has always been the core topic of Sichuan opera musical reform. The related literature conclude: Feng Guangyu, in the work Discussion of the High-pitched Tune Music of Sichuan Opera, has taken positive study and practice of Sichuan opera's high-pitched tune musical reform from the aspects of strengthening the musical beat(measure in traditional Chinese music) of vocal cavity, enriching the theatricality of high-pitched tune music, strengthening the musicality of 'one-word vocal cavity' and enriching the expressive force of aria. He Guangbiao, in the work The Key Point of Sichuan Opera High-pitched Tune Musical Reform--Aria, has pointed out that the melody of Sichuan opera's aria is not strong with more sad melody and less happy melody, which is not helpful to express people's feeling in this new era, apart from that, it should also combine tightly with Sichuan's language rhyme. Fangyin, in the work Two Difficulties and Breakthrough of Sichuan Opera Musical Reform, has expounded the innovation and development history of Sichuan 
opera's vocal cavity melody from the aspects of relation between changes and melody, inner potential of Sichuan opera's vocal cavity and absorption of external elements etc., besides, he has also put forward that it is necessary to strengthen the form-innovation awareness of vocal cavity art.

The literature taking discussion from the perspectives of singing ability and ways include: How the Sichuan Opera Actors Get with the Modern Orchestra written by Gan Shaocheng, this essay has taken the intonation of Sichuan opera pitch and Chinese character tone as the pointcut and has introduced the importance of spectrogram identification and voice training,on which basis, it has put forward that the traditional teaching method and inheritance system of oral or mental instruction should be changed completely. Pengjuan, in the Work Musical Sensibility and Rhythm Sensation of Opera Actors has put forward the master of musical sensibility and rhythm sensation comes from the deep understanding of melody tastes, lyrics meaning and emotion changing and the proper use of vocal cavity style and forms, which can be achieved only by expanding the horizon, learning culture and studying music so that to complete the procure of first knowing of, later understanding, finally application. Yu Wentong, Jiang Lifang, in the work Wonderful and Tactful Melody, Clear and Roundabout Sound, has expounded from the aspects of adding flowers to melody, lengthening and shortening of melody, tune-pattern changes, rhythm and tune-polishing etc., that the aria reform needs to inherit the past tune pattern and remove the old musical plate, in which way, can the aria achieve the effect of being both excellent in voice feeling and lyric feeling. Zeng Shufang, in the work Discussion of the Inheritance and Development of Sichuan Opera's Vocal Music and Zuo Qingfei in the work The Feasible Way--Combination of Western Music Sound Production and Chinese Opera Singing Method, have put forward that the singing of Sichuan opera should borrow from the bel canto, furthermore learn widely from others' strong points and also have a style of one's own, only in which way, can it express the emotion in lyric and express singing in emotion.

The playing of gong and drum in Sichuan opera is the important component reflecting its local characteristics and it is also the main part of Sichuan opera musical reform. The discussion developed from the aspects of gong-drum and band accompaniment include: The Inheritance and Development of Sichuan Opera's Percussion Music written by Wang Guanfu, in that essay, the author has concluded the function of percussion music in Sichuan opera, on which basis, he has put forward that the drummer should enhance advantage and avoid disadvantage, learn widely from others' advantages and sincerely cooperate with other instrumentalists, besides, the gong and drum should take reform in manufacturing craft and musical instrument property. Liu Dingyu, in the essay Reform Exploration of Sichuan Opera's Percussion Music and Shixin, in the essay Expanding of Percussion Music's Art Expressive Force have put forward that the percussion music reform should be be synchronized with the development and changes of playing methods, besides, it should develop in the direction of melodic percussion music and multiple timbre and adjust reasonably the compound mode, strong or weak hierarchy and the whole layout of gong and drum in Sichuan opera, apart from that, it also has involved the discussion of percussion instruments and effectual musical instruments reform. Pei Shouxun and Zhang Yong'an, in the essay Discussion of the Current Situation and Reconstruction of Sichuan Opera's Percussion Music, have put forward that the percussion music mechanism should be changed and the artistic concept of percussion music should be rebuilt, besides, the changing of percussion music's structure should be coadapted to the modern band and cultivating percussion music talents with modern thinking is the complex and challenging task.

This kind of research literature is detailed and complicated with large quantities, besides, various design plans and reform measures have success and failure, however, they have presented the concrete situation of Sichuan opera musical reform in different stages and fields, which is of great 
historical value.

\section{Retrospect, Conclusion and Reflection of Sichuan Opera Musical Reform}

Currently speaking, the existing literature that take conclusion and refection of the history of Sichuan opera musical reform is rare and most of the literature mainly take collating from the success and failure, gain and loss of aria and tune names etc. ontology reforms so that to conclude the experience and lessons, however, these literature lack the discussion of social and historical necessity and further reasons from the macroscopic and global perspective.

The literature that have taken retrospect and rearrangement of the Sichuan opera music reform history since the foundation of China include: He Guojing, in the essay, Historical Background and Experience of Sichuan Opera Musical Reform in the 50th , has pointed out that the contemporary Sichuan opera begins to present the trend of isolating from the people and the purpose of music design is awards and foreign visit, but not the performance in theater, as a result, some traditional and important parts have been damaged and some repertoire have lost, even that one big opera is divided into three small operas, which is the worrying problem under the current situation. Peng Chaoyi, in the essay Three Topics of Sichuan Opera Musical Reform has retrospected the achievement got in Sichuan opera musical reform from the aspects of composition skills, thinking ways and band organizational system etc., besides, he has also discussed the existing problems of Sichuan opera musical reform from the aspects of musical groups quantity decline sharply, practitioner quantity decline, neglecting of artistry, on which basis, he has put forward his own suggestion and proposition. Zhang Yong'an, in the essay A Glimpse of the Gain and Loss of Sichuan Opera Musical Reform, has pointed out that there is still a wide gap between the expressive force of Sichuan opera music and the aesthetic appreciation of times and this gap can not be filled by large number of music design for many repertory, lots of musical instruments or the quantity of orchestration, but be filled by taking study of the aesthetic appreciation characteristics, structure basis reform necessity and importance of traditional art, so as to find the solutions. Qiu Yonghe and Huang Weiyu, in the work Retrospect and Reflection of Sichuan Opera Musical Reform, have taken overall rearrangement of the Sichuan opera musical reform history since the establishing of China from the dimension of time. On the time of affirming the achievement, the disadvantages has also been concluded from the aspects of reform limitation, theory going behind the practice and lack of musical consciousness for opera types etc., furthermore, suggestion and opinions have been put forward by the author.

Throughout the above literature document, the existing literature about the development and reform of Sichuan opera musical reform in the later half of the 20th mainly take expounding and discussion only from one single aspect, such as reform measures, implementation plans or experience and lessons, besides, that discussion and expounding present the characteristics of fragmentation and fragmentation, further more, the academic researches about the integral investigation, presentation, analysis and conclusion of the historical full picture for Sichuan opera musical reform are still of few number. Under that situation, the author has suggested that on the basis of abundant field investigation and individual case interview, it is necessary to use such expression context as the combination of central policy and regional characteristics and present comprehensively the true historical process of Sichuan opera musical culture development and reform under the joint pushing of management organization, performance troupes and artists from the aspects of influence of national cultural policy on local opera art development, the role of local government agencies and important troupes in Sichuan opera musical reform and the role of Sichuan opera artistic groups in reform activities etc. Meanwhile, it also needs to give full 
consideration to the concrete factors of national policies, local characteristics, individual behavior, industrial practice and cultural psychology, so as to get the idea of the historical process of Sichuan opera musical dynamic evolution into shape, furthermore, take analysis of the unique characteristics of Sichuan opera musical reform and a series of situation and problems appearing in the reform process and then develop the in-depth study in this field.

\section{Acknowledgements}

Ministry of Education Humanities-Social Science Study Youth Fund Project 'Formation, Development and Evolution Study of Modern Sichuan Opera’, Project No. : 15YJC760123.

\section{References}

[1] Discussion of Sichuan Opera's Basic Theory (2 periods in total ), Sichuan Opera Art Academy of Sichuan Province, edited and printed in 1984.

[2] Anthology of Prospering Sichuan Opera Literary Theories, Sichuan Opera Leading group of Sichuan province, Sichuan Opera Art Academy of Sichuan Province, edited and printed in 1986.

[3] Anthology of Sichuan Opera Musical Reform Literary, Sichuan Opera Art Academy of Sichuan Province, edited and printed in 1991.

[4] Sichuan Opera Art Academy of Sichuan Province: Thinking in the New Century--Anthology of Sichuan Opera Innovative Theory Seminars in the 90th , Bashu Publishing House, 1999.

About the author: Yue Shengdong(1978.6-), Male, Doctor, Born in Yuzhong district, Chongqing province, Associate professor working in the School of Music, Chengdu Normal University

通讯地址：成都市温江区海科路东段 99 号成都师范学院音乐学院，611130 